\title{
Research Brief \\ Experience Disclosing Mental Health Conditions Among College Students from Different Ethnic Backgrounds
}

\author{
Shilpa R. Hampole, BS \\ Steven H. S. Nguyen, BS \\ Erin L. Woodhead, PhD \\ San José State University
}

\begin{abstract}
Aim: The current study compared disclosure of mental health problems to staff, faculty, and peers among college students from different ethnic backgrounds.

Background: Although there are differences in mental health stigma between college students from different ethnic backgrounds, there is limited research on whether these differences are associated with negative experiences disclosing mental health conditions on campus.

Methods: The sample $(N=66)$ was $71 \%$ female; average age was 19.03 years $(S D=1.14)$. Participants identified as Latino/a (35.4\%), Asian American (33.8\%), Caucasian (13.8\%), or other/mixed ethnicity $(16.9 \%)$.

Results: For disclosure to staff, there was a main effect of ethnicity. Post-hoc analyses found that Latino/a students were significantly more comfortable disclosing mental health problems to staff than Asian American students. There were no significant effects for disclosure to faculty or peers.

Conclusions: This preliminary study suggests that universities must optimize outreach and mental health services for different ethnic groups to improve campus experience around mental health conditions.
\end{abstract}

Submitted 7 August 2019: accepted 2 October 2019

Keywords: disclosure, mental health, college students, ethnic groups

There is increasing concern about the mental health of college students (Auerbach et al., 2018; Bruffaerts et al., 2018). As many as one in three college students screen positive for at least one mental health problem (Bruffaerts et al., 2018). Commonly reported conditions include major depression, mania, general anxiety disorder, panic disorder, and substance use disorders. In a five-year study on the state of college student mental health, Xiao et al. (2017) found that there was a significant upward trend in self-reported distress stemming from generalized anxiety, depression, and social anxiety, with a decreasing trend for substance use.

At some point, students with mental health problems may need to disclose information to faculty and staff in order to obtain campus services, or extensions on assignments for specific classes. In addition, students may want to disclose to peers in order to obtain support (Hefner \& Eisenberg, 2009). In one study, 56\% of participants reported feeling embarrassed about disclosing their condition to faculty; $56 \%$ reported a fear of being stigmatized by faculty. Other concerns included a lack of cooperation from faculty ( $42 \%$ of participants) and a fear of being stigmatized by 
peers (41\% of participants; Salzer, Wick, \& Rogers, 2008). This and other research suggest that disclosure of mental health problems is highly stressful for students (Barnard-Brak, Lectenberger, \& Lan, 2010) and is influenced by personal beliefs and stigma around mental health.

The increasing ethnic diversity of college students (Public Policy Institute of California, 2017) means that students bring beliefs from their own culture to campus, including beliefs about the nature of mental health problems and stigma around mental health. Asian American college students tend to be the lowest users of mental health services, followed by African American students and then Latino/a students (Cheng, Kwan, \& Sevig, 2013). In the Cheng et al. (2013) study, Asian American students scored higher on self-stigma and perceived stigma from family and friends than African and Latino American students. In another study on stigma among college students, Wu et al. (2017) found that Asian American students were more likely to report high self-stigma and high public stigma around mental health, which was associated with lower use of mental health services. Although the research presented here informs our understanding of cultural differences in mental health stigma among college students, there is a need to take this research one step further to understand students' experiences when they decide to discuss or disclose mental health problems with staff, faculty, or peers.

In light of the research suggesting that mental health stigma varies significantly across cultures (Cheng et al., 2013; Wu et al., 2017), the current study compared the disclosure experiences of college students from different ethnic backgrounds. We hypothesized that Asian American college students would report worse experiences than other ethnic groups, consistent with the research presented above. Although this study is preliminary, comparison of disclosure experiences across ethnic groups helps campuses better understand the experiences of mental health disclosure among historically under-represented groups.

\section{METHODS}

\section{Participants}

The participants for the current study $(n=66)$ were part of a larger study $(N=190)$ on learning and neurological differences among college students. The current study included participants who reported mental health conditions only, without comorbid physical conditions or learning differences. Five students were registered for accommodations with the campus accessibility center. Participants were eligible for the study if they were age 18 and over and selfidentified as having a mental health problem that impacted their academics. Types of conditions included posttraumatic stress disorder (PTSD), depression, bipolar disorder, panic disorder, social anxiety, generalized anxiety disorder, and test-taking anxiety. Participants also reported if they had been formally diagnosed (yes $=40.6 \%$ ). The majority of participants were female $(72.3 \%)$ with an average age of 19.03 years $(S D=1.14$, range 18 - 23). Participants identified as Hispanic/Latino (36.4\%), Asian (31.8\%), Caucasian (16.7\%), South East Asian (9.1\%), Native Hawaiian or Pacific Islander (4.5\%), Black/African-American (3.0\%), American Indian or Alaskan Native (1.5\%) or Middle Eastern or North African (1.5\%).

\section{Measures}

Students with Accommodation Needs Satisfaction Survey (SWANSS). The SWANSS is a 38-item online selfreport survey that has established reliability and validity (Smith, Woodhead, \& Chin-Newman, 2019). Content validity 
was established through interviews with students receiving accommodations, as well as pilot testing with faculty and staff. Reliability for the non-demographic items was .90 (Smith et al., 2019). Question topics include: (1) background information on the problem/condition (preferred terms, age and location of diagnosis), (2) accommodation use, if registered with the campus accessibility center, (3) disclosure and visibility of conditions, (4) satisfaction scales regarding disclosure to staff, faculty, and peers, and (5) general demographics. For the satisfaction scales, means were calculated for each subscale: five items for experience disclosing to faculty (i.e., My professors are respectful when I disclose; Cronbach's alpha $=.92$ ); three items for experience disclosing to staff (i.e., Staff members respond positively when I ask for accommodations or discuss my condition; alpha $=.88)$, and three items experience disclosing to peers (i.e., I feel comfortable discussing my accommodation needs or condition with other students; alpha $=.89$ ). Rating scales from 1 to 10 were used throughout the survey.

\section{Procedure}

Participants were recruited via emails from the campus accessibility center which included the study link and a brief description of the study. Two emails were sent, one in the middle of the semester and one toward the end of the semester. No additional compensation was provided to participants who were registered with the campus accessibility center. Participants were also recruited via an online system available to students taking Psychology classes. In this online system, students could sign up for studies and receive course credit for participating in the survey if their psychology course required research participation. An alternative writing assignment was available for students who did not want to participate in research studies. All participants completed the survey online through Qualtrics. Study approval was obtained from the Institutional Review Board at San José State University.

\section{Analysis plan}

Three factorial ANOVAs $(4 \times 2 \times 2)$ were conducted to assess the impact of ethnicity, gender, and formal diagnosis on disclosure experiences to staff, faculty, and peers. Tukey tests were used for post-hoc analyses comparing the different ethnic groups. We controlled for gender and presence of a formal diagnosis (yes or no) for any of the conditions. For the purposes of the study, we created a group of mixed/other ethnicity, which included small sample size groups (Middle Eastern or North African, Black/African-American, American Indian or Alaskan Native, South East Asian, and Native Hawaiian or Pacific Islander) and participants who endorsed more than one of the presented ethnicity categories $(n=5)$. All analyses were conducted in SPSS version 25; the significance level was set at 0.05.

\section{RESULTS}

For disclosure to staff, there was a significant main effect of ethnicity (Table 1). Post-hoc analyses found that Latino/a students $(M=6.72, S D=2.34)$ were more comfortable disclosing to staff than Asian students $(M=4.03, S D=2.30$; $p=.006$ ). All other post-hoc comparisons for staff disclosure were not significant (Caucasian $M=5.17, S D=2.88$; Mixed/Other $M=6.10, S D=2.67)$. There were no significant main effects for disclosure to faculty or peers. 
Table 1

ANOV A Results for Disclosure to Faculty, Staff, and Peers

\begin{tabular}{llllllll}
\hline Target & Predictor & $\begin{array}{l}\text { Sum of } \\
\text { Squares }\end{array}$ & & $\begin{array}{l}\text { Mean } \\
\text { Square }\end{array}$ & $F$ & $p$ & $\begin{array}{l}\text { Partial } \\
\eta 2\end{array}$ \\
\hline Staff & (Intercept) & 1300.55 & 1 & 1300.55 & 211.35 & .000 & .80 \\
& Ethnicity & 63.32 & 3 & 21.11 & 3.43 & $.023^{*}$ & .16 \\
& Gender & 2.02 & 1 & 2.02 & .33 & .569 & .01 \\
& Diagnosis & 4.89 & 1 & 4.89 & .79 & .377 & .02 \\
Faculty & (Intercept) & 1154.75 & 1 & 1154.75 & 180.74 & .000 & .77 \\
& Ethnicity & 43.99 & 3 & 14.66 & 2.30 & .088 & .11 \\
& Gender & .94 & 1 & .94 & .15 & .703 & .00 \\
& Diagnosis & 23.17 & 1 & 23.17 & 3.63 & .062 & .06 \\
Peers & (Intercept) & 1300.76 & 1 & 1300.76 & 199.86 & .000 & .80 \\
& Ethnicity & 8.36 & 3 & 2.79 & .43 & .734 & .03 \\
& Gender & 5.09 & 1 & 5.09 & .78 & .381 & .02 \\
& Diagnosis & .02 & 1 & .02 & .00 & .955 & .00 \\
\hline
\end{tabular}

${ }^{*} p<.05$

\section{DISCUSSION}

The results of the current study support existing research on mental health stigma among college students, particularly that Asian American college students report more stigma than other ethnic groups (Cheng et al., 2013; Wu et al., 2017). The significance of the current study is that these ethnic group differences also extend to experiences disclosing mental health conditions to staff.

It is particularly concerning that Asian American students reported negative experiences disclosing to staff compared to other ethnic groups. Campus staff are often the initial contacts for obtaining resources either through accommodations or other types of resources on campus, such as student health and wellness centers. College students want more information about school/work/life balance and stress management (Gibbons et al., 2019), and may turn to campus staff in order to obtain this information. Some unique college programs have involved faculty and staff in sharing stories about their experiences as first-generation college students (King, Griffith, \& Murphy, 2017). Similar types of programs may be effective for stress and mental health problems on campus. 
Staff and faculty may also need additional training on how to interact with students who are talking about mental health problems. These types of trainings have been developed for non-mental health professionals who come into contact with people with mental health problems (e.g., police, teachers; Booth et al. 2017) and could be adapted to meet the needs of campus staff.

A limitation of the current study is that participants did not need a formal diagnosis in order to participate in the study. Additionally, we did not verify diagnoses if participants reported that they received a formal diagnosis at some point. We chose to include conditions that were either formally diagnosed or not formally diagnosed due to the multiple reasons why students may not seek a formal diagnosis, including mental health stigma and lack of access to mental health care (Burton Denmark, Hess, \& Swanbrow Becker, 2012). The results could have been different if we focused only on participants who were receiving accommodations, or only participants who had a formal diagnosis.

Despite the limitations of the current study, the results suggest that targeted outreach is needed to understand how to meet the needs of Asian American college students with mental health conditions. Future research should focus on how to create campus climates that foster discussion of mental health problems, both within and across cultures. 


\section{REFERENCES}

Auerbach, R. P., Mortier, P., Bruffaerts, R., Alonso, J., Benjet, C., Cuijpers, P., .. Kessler, R. C. (2018). WHO World Mental Health Surveys International College Student Project: Prevalence and distribution of mental disorders. Journal of Abnormal Psychology, 127, 623-638. (Supplemental) https://doi.org/10.1037/abn0000362.supp

Barnard-Brak, L., Lectenberger. D., Lan, W. Y. (2010). Accommodation strategies of college students with disabilities. The Qualitative Report, 15, 411-429.

Booth, A., Scantlebury, A., Hughes-Morley, A., Mitchell, N., Wright, K., Scott, W., \& McDaid, C. (2017). Mental health training programmes for non-mental health trained professionals coming into contact with people with mental ill health: A systematic review of effectiveness. BMC Psychiatry, 17, 196. https://doi.org/10.1186/s12888-017-1356-5

Burton Denmark, A., Hess, E., \& Swanbrow Becker, M. (2012). College students' reasons for concealing suicidal ideation. Journal of College Student Psychotherapy, 26, 83-98.

Bruffaerts, R., Mortier, P., Kiekens, G., Auerbach, R. P., Cuijpers, P., Demyttenaere, K.,... Kessler, R., C. (2018) Mental health problems in college freshman: Prevalence and academic functioning. Journal of Affective Disorders, 225, 97-103. https://doi.org/10.1016/j.jad.2017.07.044

Cheng, H.-L., Kwan, K.-L. K., \& Sevig, T. (2013). Racial and ethnic minority college students' stigma associated with seeking psychological help: Examining psychocultural correlates. Journal of Counseling Psychology, 60, 98 111. https://doi.org/10.1037/a0031169

Gibbons, S., Trette-McLean, T., Crandall, A., Bingham, J. K., Garn, C. L., \& Cox, J. C. (2019). Undergraduate students survey their peers on mental health: Perspectives and strategies for improving college counseling center outreach. Journal of American College Health. https://doi.org/10.1080/07448481.2018.1499652

Hefner, J., \& Eisenberg, D. (2009). Social support and mental health among college students. American Journal of Orthopsychiatry, 79, 491-499. https://doi.org/10.1037/a0016918

King, C., Griffith, J., \& Murphy, M. (2017). Story sharing for first-generation college students attending a regional comprehensive university: Campus outreach to validate students and develop forms of capital. TeacherScholar: The Journal of the State Comprehensive University, 8, Article 1.

Public Policy Institute of California. (2017). Higher education in California: Increasing equity and diversity. Retrieved from https://vtechworks.lib.vt.edu/bitstream/handle/10919/83673/IncreasingEquityDiversity.pdf?sequence=1 \&isAllowed $=\mathrm{y}$

Salzer, M.S., Wick, L.C., \& Rogers, J. A. (2008). Familiarity with and use of accommodations and supports among postsecondary students with mental illnesses. Psychiatric Services. 59, 370-375. https://doi.org/10.1176/ps.2008.59.4.370

Smith, S. A., Woodhead, E., \& Chin-Newman, C. (2019). Disclosing accommodation needs: Exploring experiences of higher education students with disabilities. International Journal of Inclusive Education. https://doi.org/10.1080/13603116.2019.1610087 
Wu, I. H. C., Bathje, G. J., Kalibatseva, Z., Sung, D., Leong, F. T. L., \& Collins-Eaglin, J. (2017). Stigma, mental health, and counseling service use: A person-centered approach to mental health stigma profiles. Psychological Services, 14, 490-501. https://doi.org/10.1037/ser0000165

Xiao, H., Carney, D. M., Youn, S. J., Janis, R. A., Castonguay, L. G., Hayes, J. A., \& Locke, B. D. (2017). Are we in crisis? National mental health and treatment trends in college counseling centers. Psychological Services, 14, 407-415. https://doi.org/10.1037/ser0000130

Author correspondence may be addressed to:

Erin L. Woodhead, PhD

Associate Professor

Department of Psychology

San José State University

San José, CA 95192-0120

Erin.Woodhead@sjsu.edu

\section{Author's Note and Acknowledgements}

A version of this paper was presented at the 2019 annual meeting of the Western Psychological Association. This work was supported by a Faculty Diversity Development Research, Scholarship and Creative Activities Award from the Office of Diversity, Equity \& Inclusion at San José State University, awarded to Erin Woodhead. 